\title{
NEONATAL EARLY ONSET SEPSIS: EFFICIENT INTERDISCIPLINARY COMMUNICATION ESSENTIAL TO SAFE PRACTICE
}

\author{
Dr Sindugaa Sivasubramaniam - ST3 Paediatrics, Dr Rebecca Williams - ST2 Paediatrics \\ Supervisor - Dr Venkat Thiyagesh - Consultant Paediatrician
}

\section{BACKGROUND}

Early-onset neonatal sepsis (onset within 72 hours of birth) is a significant cause of mortality and morbidity in newborns. The incidence is about $2 / 1000$ live births with mortality rates approximately $10-15 \%$ for these infants. The most common pathogens are Group B haemolytic Streptococcus (GBS), E. Coli and Listeria monocytogenes. GBS sepsis can be especially severe in newborns, with over $90 \%$ of GBS sepsis presenting within 12 hours of delivery. This highlights the importance of prompt recognition and treatment of at risk babies.

NICE Guidelines CG149 on the prevention and treatment of early neonatal sepsis clearly states that 'if a baby needs antibiotic treatment it should be given as soon as possible and always within 1 hour of the decision to treat'. Yet, despite this, it is commonly reported that there are delays from making the decision to commence treatment to the delivery of antibiotics on the wards.

\section{OBJECTIVE}

1. To audit compliance with NICE guidelines CG149 on timely antibiotic administration on the postnatal wards and delivery unit at a district general hospital.

2. To improve the detection of at-risk patients and facilitate prompt antibiotic administration within the recommended golden hour

\section{METHODS}

Over a 4 week period we prospectively collected data on babies started on antibiotics on the postnatal wards. The primary outcome was whether these babies received antibiotics within the 1 hour of the decision being made. Reasons for any delays were noted.

\section{Calderdale and Huddersfield}

\section{NEONATAL SEPSIS}

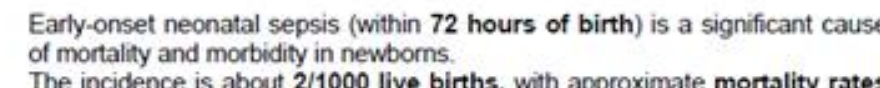
of mortality and morbidity in newborms
The incidence is about $2 / 1000$ live births, with approximate mortality rates The incidence is about 2211 so
of $10.15 \%$ for these infants

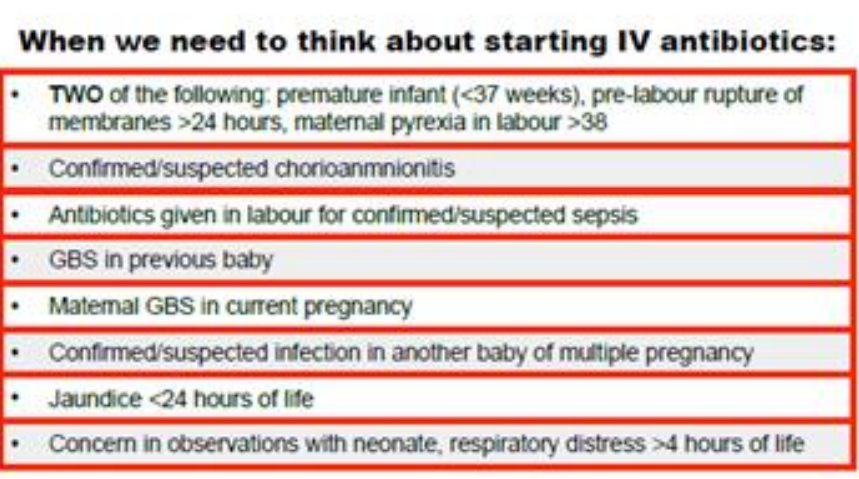

\section{THE GOLDEN HOUR}

NICE Guidelines say - If a baby needs antibiotic treatment it should be given as soon as possible and always within 1 hour
of the decision to treat.

Contact Paeds SHO \#6677 or Reg \#2130

Figure 1. Neonatal Sepsis Awareness Poster

\section{RESULTS}

Results illustrated that there was significant difference in outcomes following intervention (Figure 2):

Initially only $19 \%$ of patients received their antibiotics within the recommended golden hour, however, following team education measures this increased to $61 \%$.

Late administration of antibiotics by postnatal staff initially contributed to $62 \%$ of delays, despite timely cannulation. This then improved to $31 \%$.

Identification of neonates needing antibiotics by the midwifery staff and communication of this to the neonatal team remained unchanged despite intervention (negligible improvement of $2 \%$ ) Delays in obtaining IV access improved from 19\% to $6 \%$

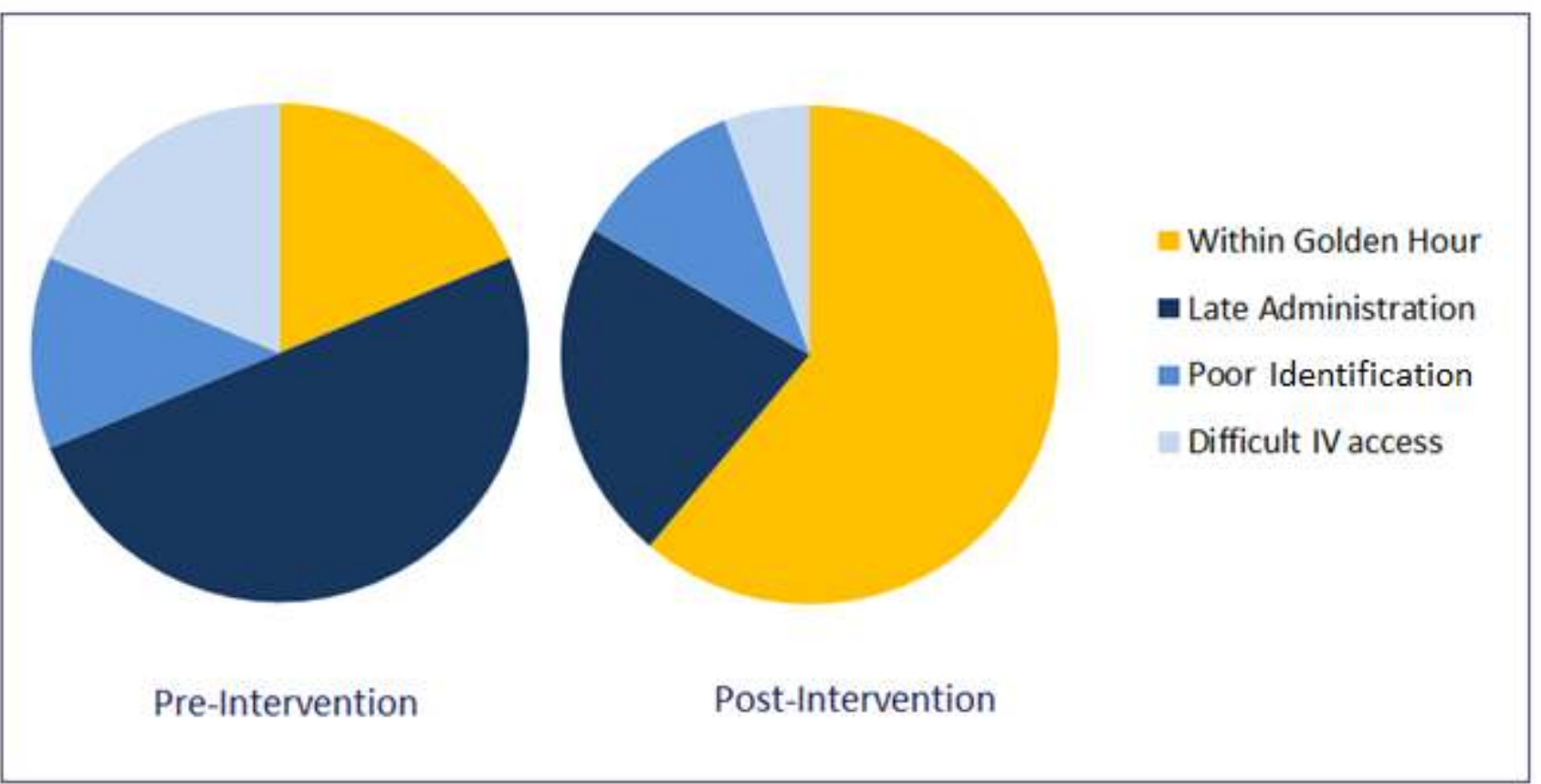

Figure 2. Proportion of patients receiving antibiotics within the recommended golden hour and the factors contributing to delays both before and after intervention

\section{CONCLUSION}

The first audit cycle highlighted poor compliance with NICE guidelines CG149. However, following the education of team members in the importance of timely identification of patients and the communication of this information between different departments, we saw a significant improvement in at risk neonates receiving antibiotics within the recommended golden hour.

We initially did not expect to see an improvement in cases where difficulties in obtaining intravenous access led to delays. However, as demonstrated above, by raising awareness of an area in need of improvement there was faster escalation in situations of failed cannulation. This further demonstrates the ability of efficient and effective communication to enhance patient outcomes.

Unfortunately, timely identification of neonates needing antibiotics has not improved. Identifying and breaking down barriers to this will be the focus of the next audit cycle.

\section{REFERENCES}

National Institute for Health and Care Excellence (2016). Neonatal infection (early onset): antibiotics for prevention and treatment. (Clinical guideline CG149). Retrieved from: 